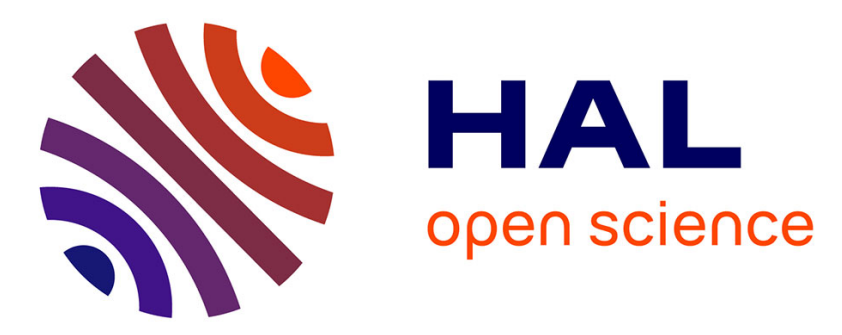

\title{
Spectral statistics of Hamiltonian matrices in tridiagonal form
}

\author{
R. A. Molina, A.P. Zuker, A. Relaño, J. Retamosa
}

\section{To cite this version:}

R. A. Molina, A.P. Zuker, A. Relaño, J. Retamosa. Spectral statistics of Hamiltonian matrices in tridiagonal form. Physical Review C, 2005, 71, 064317 (5 p.). 10.1103/PhysRevC.71.064317 . in2p300024568

\section{HAL Id: in2p3-00024568 https://hal.in2p3.fr/in2p3-00024568}

Submitted on 1 Jun 2021

HAL is a multi-disciplinary open access archive for the deposit and dissemination of scientific research documents, whether they are published or not. The documents may come from teaching and research institutions in France or abroad, or from public or private research centers.
L'archive ouverte pluridisciplinaire HAL, est destinée au dépôt et à la diffusion de documents scientifiques de niveau recherche, publiés ou non, émanant des établissements d'enseignement et de recherche français ou étrangers, des laboratoires publics ou privés. 


\title{
Spectral statistics of Hamiltonian matrices in tridiagonal form
}

\author{
R. A. Molina, ${ }^{1,2}$ A. P. Zuker, ${ }^{3}$ A. Relaño, ${ }^{4}$ and J. Retamosa ${ }^{4}$ \\ ${ }^{1}$ Commissariat a l'Energie Atomique, Service de Physique de l'Etat Condensé, F-91191 Gif-sur-Yvette, France \\ ${ }^{2}$ Max-Planck-institut für Physik Komplexer Systeme, Nöthnitzer Strasse 38, D-01187 Dresden, Germany \\ ${ }^{3}$ IReS, Bât27, IN2P3-Centre National de la Recherche Scientifique/Université Louis Pasteur BP 28, F-67037 Strasbourg Cedex 2, France \\ ${ }^{4}$ Departamento de Física Atómica, Molecular y Nuclear, Universidad Complutense de Madrid, E-28010 Madrid, Spain
}

(Received 1 April 2004; revised manuscript received 13 December 2004; published 27 June 2005)

\begin{abstract}
When a matrix is reduced to Lanczos tridiagonal form, its matrix elements can be divided into an analytic smooth mean value and a fluctuating part. The next-neighbor spacing distribution $P(s)$ and the spectral rigidity $\Delta_{3}$ are shown to be universal functions of the average value of the fluctuating part. It is explained why the behavior of these quantities suggested by random matrix theory is valid in far more general cases.
\end{abstract}

DOI: 10.1103/PhysRevC.71.064317

PACS number(s): 21.10.Dr, 05.45.Mt, 24.60.Lz

\section{INTRODUCTION}

The core of random matrix theory (RMT) is a corpus of exact results concerning the canonical Gaussian ensembles of matrices, invariant under three types of transformations: orthogonal Gaussian orthogonal ensemble (GOE), unitary Gaussian unitary ensemble (GUE), and symplectic Gaussian symplectic ensemble (GSE). The matrix elements are, respectively, real, complex, and quaternionic numbers normally distributed. The pioneering papers by Wigner, Dyson, Porter, Gaudin, Metha, and others are collected in [1]. Extensions to other ensembles are reviewed in [2], but there are few exact results for them, and numerical simulations become the rule. One important exception is the work of Mon and French, which shows how the level densities evolve from a semicircle law for GOE to a Gaussian one as the number of particles increase for many-body random Hamiltonians [3]. In general, such Hamiltonians - and a fortiori nonrandom ones-have properties that differ from those of the canonical ensembles. Their study usually proceeds by the splitting of the Hamiltonian as $H=H_{d}+\lambda V$, where $H_{d}$ is diagonal in some "natural" (e.g., mean field) basis and $V$ induces mixing, and then the evolution of the system is followed as a function of $\lambda$. Some authors do it through quantities that depend on the wave functions - such as information entropy or number of principal components - and offer good physical insight but are basis and Hamiltonian dependent [4-6]. The widely followed alternative relies on the fluctuation properties of the spectrum, in particular the nearest-neighbor spacing distribution $P(s, \beta)$, where $s$ is the difference in energy of two consecutive levels in units of the local average spacing and $\beta$ is a repulsion parameter. In its original form, the Wigner surmise

$$
P(s, \beta)=a_{\beta} s^{\beta} \exp \left(-b_{\beta} s^{2}\right)
$$

is valid for GOE, GUE, and GSE for $\beta=1,2$, and 4, respectively. The quantities $a_{\beta}$ and $b_{\beta}$ are normalization constants fixed through the condition that the integral of $P(s)$ and the mean level spacings be unity. The small spacing behavior of this distribution is given by

$$
P(s, \beta) \sim s^{\beta} \quad \text { for } \quad s \ll 1,
$$

which defines the repulsion parameter $\beta$. One should note that the values of $\beta$ that correspond to the exact $P(s)$ for the canonical ensembles [1] may not be that of Eq. (1), which is only a good approximation. To give an example: The exact GOE result corresponds to $\beta=0.957$. To adapt it to $H=H_{d}+\lambda V$ studies (usually restricted to real-symmetric matrices) it is necessary to find forms to extrapolate between the Poisson law $P(s, 0) \approx \exp -s$ characteristic of $(\lambda=0)$ integrable systems [7] and the GOE limit, soon reached for increasing $\lambda$ and characterized by a $P(s)$ very close to the Wigner surmise, $P(s, 1)=(\pi s / 2) \exp \left(-\pi s^{2} / 4\right)$. Two standard forms are used to fit $P(s, \beta)$ for a given $\lambda$, Brody's [8];

$$
\begin{aligned}
P_{B}(s, \beta) & =\alpha(\beta+1) s^{\beta} \exp \left(-\alpha s^{\beta+1}\right), \\
\alpha & =\left(\Gamma\left[\frac{\beta+2}{\beta+1}\right]\right)^{\beta+1},
\end{aligned}
$$

and Izrailev's [9];

$P_{I}(s, \beta)=A_{\beta} s^{\beta} \exp \left[-\frac{\pi^{2} \beta}{16} s^{2}-\left(B_{\beta}-\frac{\pi \beta}{4}\right) s\right]$,

where $A_{\beta}$ and $B_{\beta}$ are normalization constants fixed again through the condition that the integral of $P(s)$ and the meanlevel spacings be unity. The advantage of this form is that it is valid from the $\beta=0$ (Poisson) to the $\beta=\infty$ (uniform spacing, "picket-fence") limits and can be viewed as a generalization of RMT. The behavior at small spacings is the same for both distributions and allows the identification of $\beta$ with the repulsion parameter. In the Poisson-GOE interval Brody's and Izrailev's parametrizations are close, but the former does not extrapolate well to large $\beta$.

Nearest-neighbor spacing distributions measure the shortrange correlations in the spectrum. To obtain information about the long-range correlations we also calulate the spectral rigidity,

$$
\Delta_{3}(a, L)=\frac{1}{L} \min _{A B} \int_{a}^{a+L}[N(E)-A E-B]^{2} d E,
$$

which explores long-range correlations by measuring the deviations of the spectrum from a truly equidistant one. $\bar{\Delta}_{3}(L)$ is the mean value over all intervals of the same length [10]. The $\bar{\Delta}_{3}(L)$ statistic is linear for a Poisson spectrum, logarithmic 


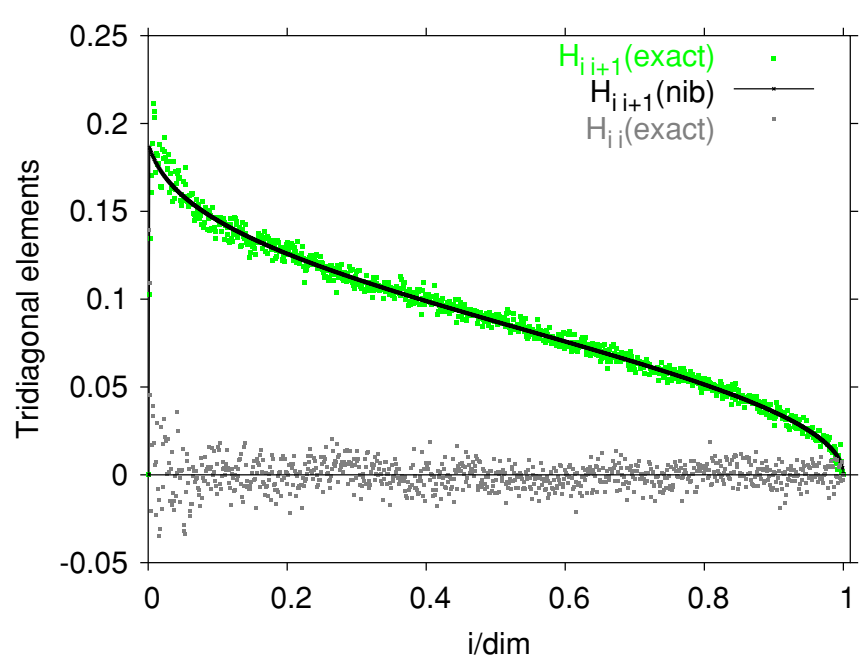

FIG. 1. (Color online) Example of the matrix elements of a Lanczos matrix for $H$ in Eqs. (8) and (9), with $e_{r}=0.07, u_{r}=y_{r}=$ $1.00, y_{c}=0 ; N=12, m=6, d=924$.

for the canonical RMT ensembles, and constant for a locally equidistant spectrum.

Although the suggestions of RMT are irrelevant for many quantities, such as level densities and information entropy, they hold true for $P(s, \beta)$. In particular, the available data on nuclear spectra follow the Wigner law [11], a doubly significant fact because (a) nuclear Hamiltonians are not random, and (b) they are dominated by the integrable mean field $H_{d}$ [which naively suggests a $P(s)$ closer to Poisson]. (Some possible counterexamples are investigated in $[12,13]$.)

Our purpose is to exhibit the general origin of fluctuation properties by splitting the Hamiltonian in a way that yields a simple universal relationship between $\beta$ and a new parameter $F$, which is subsequently introduced. The idea is that the Lanczos tridiagonal reduction of a Hamiltonian matrix leads to a canonical form for the matrix elements that are given, within fluctuations, by the four lowest moments of $H$. The off-diagonals follow an inverse binomial law (nib), whereas the diagonals are constant or more generally logarithmic [14], as illustrated in Fig 1. These smooth forms, which can be viewed as ensemble averages, define a matrix $H^{s}$, and the fluctuating part is given by $H-H^{s}$. The new strategy amounts to diagonalizing

$$
H_{i j}^{F}=H_{i j}^{s}+F\left(H_{i j}-H_{i j}^{s}\right)
$$

as a function of $F$ and at each stage extract $\beta$.

We have chosen three cases: a spin Hamiltonian, a shellmodel matrix, and a GOE one, all having $\beta=1$ at $F=1$. A universal law emerges:

$$
\beta=\frac{1}{F^{2}} .
$$

In establishing it, we will discover why RMT fluctuation properties are also universal.

In Sec. II the spin model is introduced as a generic example of Lanczos matrices. It allows a simple analysis of the role of coherence. Section III explains how to extract the $P(s, \beta)$ distributions from the spectra, the advantage of doing so with
Lanczos matrices, and the reasons for prefering Izrailev's parametrization.

\section{LANCZOS MATRICES IN A SPIN MODEL. THE ROLE OF COHERENCE}

To explain how Eq. (7) comes about, let us start with an updated summary of [14] concerning Lanczos matrices at fixed quantum numbers. This condition guarantees no subblock decomposition and hence level repulsion. The canonical characterization of the smooth part of $H$ holds for all tridiagonal matrix elements $H_{i i}$ and $H_{i i+1}$, except for small values of $i \lesssim \ln d$, where $d$ is the dimensionality of the matrix. For a number of particles $m \lesssim 5$ the off-diagonals move slowly to the $m=2$ GOE limit. Then, $\left.H_{i i+1}=\sqrt{(} 1-i / d\right)$.

These conjectures were suggested by the study of nuclear shell-model matrices, but they apply more generally. As we are interested in assessing the influence of coherence effects, it is easiest to introduce them through a Hamiltonian of $N$ spins,

$$
H=\sum_{i, i<j}\left[\varepsilon_{i} S_{i}^{0}+u_{i j} S_{i}^{0} S_{j}^{0}+y_{i j}\left(S_{i}^{+} S_{j}^{-}+S_{i}^{-} S_{j}^{+}\right)\right],
$$

where $S_{i}^{+}=u_{i}^{+} d_{i}, S_{i}^{-}=d_{i}^{+} u_{i}, S^{0}=u_{i}^{+} u_{i}-d_{i}^{+} d_{i}$ obey SU(2) commutation rules, and $u, u^{+}, d, d^{+}$are the usual fermion annihilation and creation operators. For $m$ spins up, $S^{0}=$ $\sum_{i} S_{i}^{0}=2 m-N$ is a conserved quantum number. $H$ can also be interpreted as a general pairing problem for $m$ pairs.

$$
\varepsilon_{i}=e_{r} r_{i}^{\varepsilon}, \quad u_{i j}=\frac{1}{N} u_{r} r_{i j}^{u}, \quad y_{i j}=\frac{1}{N}\left[y_{c}+y_{r} r_{i j}^{y}\right]
$$

where the random numbers $r_{i}^{\varepsilon}$ are uniformly distributed in the interval $[-0.5,0.5]$, and $r_{i j}$ are normally distributed with $\sigma \approx 0.09$.

The parameters $e_{r}$ and $u_{r}$ are associated with operators that are diagonal in the basis $\left(H_{d}\right)$, i.e., the separable part of $H$, which can be viewed as a mean field by setting

$$
\sum_{i j} u_{i j} S_{i}^{0} S_{j}^{0}=\sum_{i}\left[\sum_{j} u_{i j} S_{j}^{0}\right] S_{i}^{0} \equiv \sum_{i} \epsilon_{i} S_{i}^{0} .
$$

The coherent contribution is represented by $y_{c}\left(S^{+} S^{-}+\right.$ $S^{-} S^{+}$), where $S^{ \pm}=\sum_{i} S_{i}^{ \pm}$are the total-spin operators. This term can be interpreted as the classic schematic pairing.

The typical tridiagonal behavior for $y_{c}=0$ - shown in Fig. 1 for the set of parameters we shall use later-will evolve as $y_{c}$ increases, at first leading to logarithmic diagonals (as in Fig. 3 in [14]). Eventually $S$ will become an approximately conserved quantum number, thus violating our assumption that the matrix has no subblock decompositions: The tridiagonal elements in Fig. 1 will split in badly fluctuating pieces.

This evolution can be followed in Fig. 2 for the cumulated level density $N(E)$. For moderate values of $\left|y_{c}\right|$ the level density remains binomial, a behavior that persists as long as $\gamma_{1}^{2} / \gamma_{2}<1$, where $\gamma_{1}$ is the skewness of the level density and $\gamma_{2}$ is its excess (or kurtosis) [15]. When the third and fourth moments of the Hamiltonian violate this condition no binomial can be constructed. An interesting update of [14] is that for some large, but not too large, values such as $y_{c}=-0.25$ in 


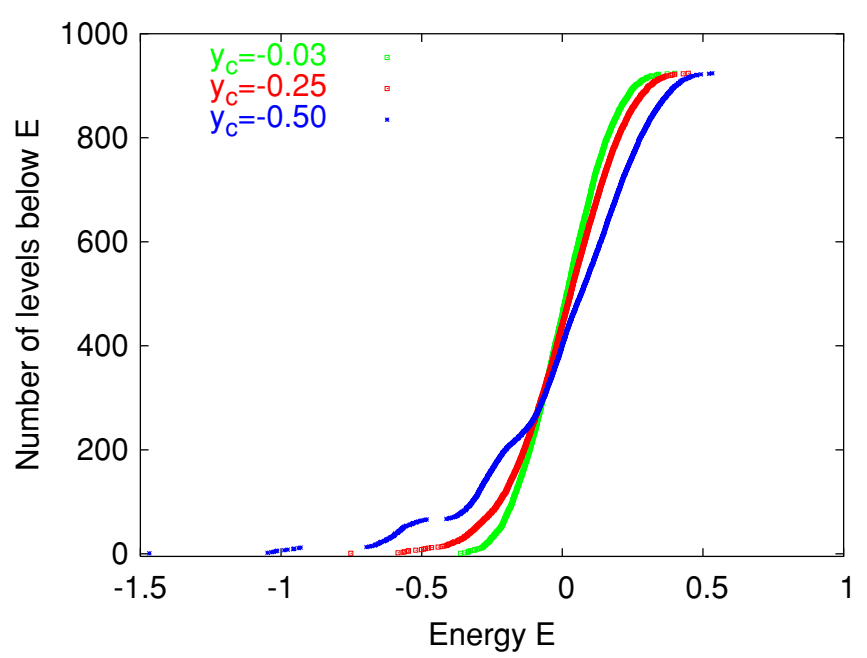

FIG. 2. (Color online) Cumulated level densities for different values of $y_{c}$ in Eqs. (9). There are well-detached ground-state points at about $E=-0.7$ for $y_{c}=-0.25$ and $E=-1.5$ for $y_{c}=-0.5$.

Fig. 2 the nib and log forms still work well in spite of the fact that the level density is no longer binomial. When $\left|y_{c}\right|$ becomes truly large, the low-lying spectrum splits in pieces of good total spin (or seniority). It is worth noting the existence of a region where the ground state is well separated but the bulk of the spectrum remains binomial.

The advantage of using random matrix elements is that small diagonalizations can be assumed to become representative of the large ones associated to realistic conditions. As Fig. 2 makes clear, the role of $y_{c}$ is to set limits to the plausibility of this assumption: When $y_{c}=-0.03$ it is easy to guess what will happen for large $N-$. When $y_{c}=-0.5$ it is a major challenge. Nonetheless, the preceding arguments indicate that substantial coherence can be absorbed by the canonical Lanczos forms.

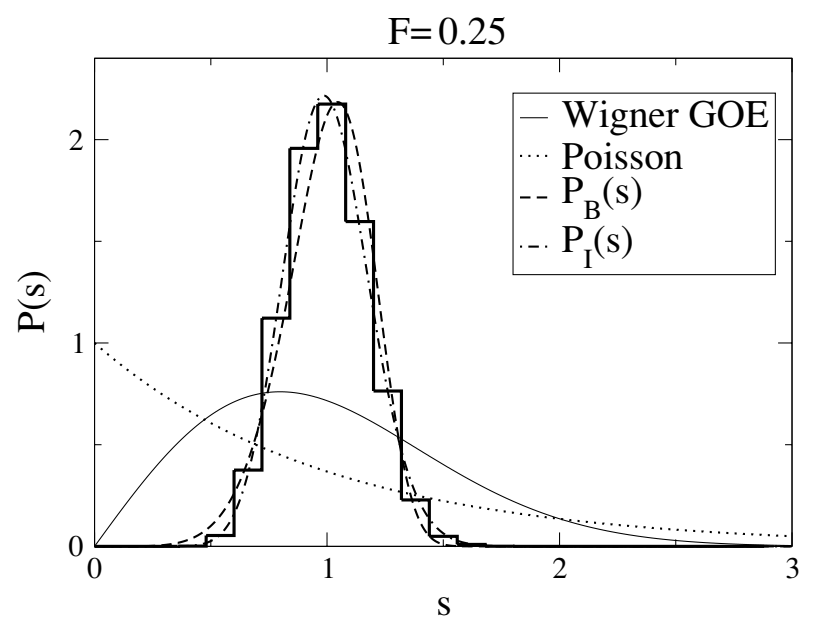

\section{FLUCTUATIONS PROPERTIES OF THE SPECTRA AND THE LANCZOS MATRIX}

To analyze fluctuation properties, the energy eigenvalues $E_{i}$ must be mapped into a dimensionless spectrum $\epsilon_{i}$ with unit local mean level spacing. This is usually accomplished through

$$
E_{i} \longrightarrow \epsilon_{i}=\bar{N}\left(E_{i}\right) \longrightarrow s_{i}=\epsilon_{i+1}-\epsilon_{i},
$$

where $\bar{N}(E)$ is the cumulated mean level density (as shown in Fig. 2) and $s_{i}$ is the variable in $P(s, \beta)$. The procedure is called unfolding, and it can be quite delicate whenever $\bar{N}(E)$ must be extracted from the numerical spectrum [16,17]. It becomes simple if one knows the analytic form of the level density, which is the case for GOE (Wigner's semicircle law) and for matrices having a binomial density

$$
\begin{aligned}
\rho(E)= & p^{(N E / S)}(1-p)^{N(1-E / S)} d \frac{N}{S} \\
& \times \frac{\Gamma(N+1)}{\Gamma(N E / S+1) \Gamma[N(1-N E / S)-1]},
\end{aligned}
$$

where $S$ is the spectrum span, $p$ is the asymmetry parameter equal to $1 / 2$ if the density is symmetric with respect to its centroid, $N$ is the effective number of particles, and $d$ is the dimensionality of the space. The way to compute these parameters as functions of the first four moments of the Hamiltonian is explained in [15]. Then $\bar{N}(E)$ becomes a well-defined smooth function $N^{S}$. As explained in Sec. II, there are situations in which the level density ceases to be a binomial, but the smooth Lanczos matrices still can be constructed with the four lowest moments. As they amount to integrable systems, they have picket-fence spectra, and the eigenvalues $E_{i}^{S}$ can be directly used to provide

$$
s_{i}=\frac{E_{i+1}-E_{i}}{E_{i+1}^{S}-E_{i}^{S}}=N^{S}\left(E_{i+1}\right)-N^{S}\left(E_{i}\right),
$$

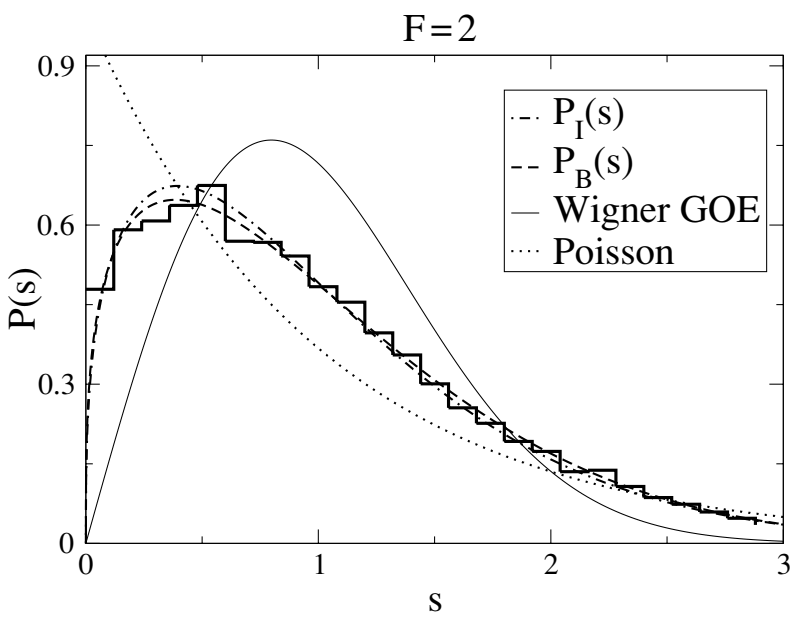

FIG. 3. Two examples of the ensemble-averaged $P(s)$ distributions and the best fit for the Brody and Izrailev distributions for the cases discussed in the text. The left-hand panel has $F=0.25$, in this case $\beta>1$, and the Izrailev distribution fits much better than the Brody distribution the numerical $P(s)$, describing correctly the behavior for small and large $s$. The right-hand panel has $F=2, \beta<1$, and both distributions do a good job. 

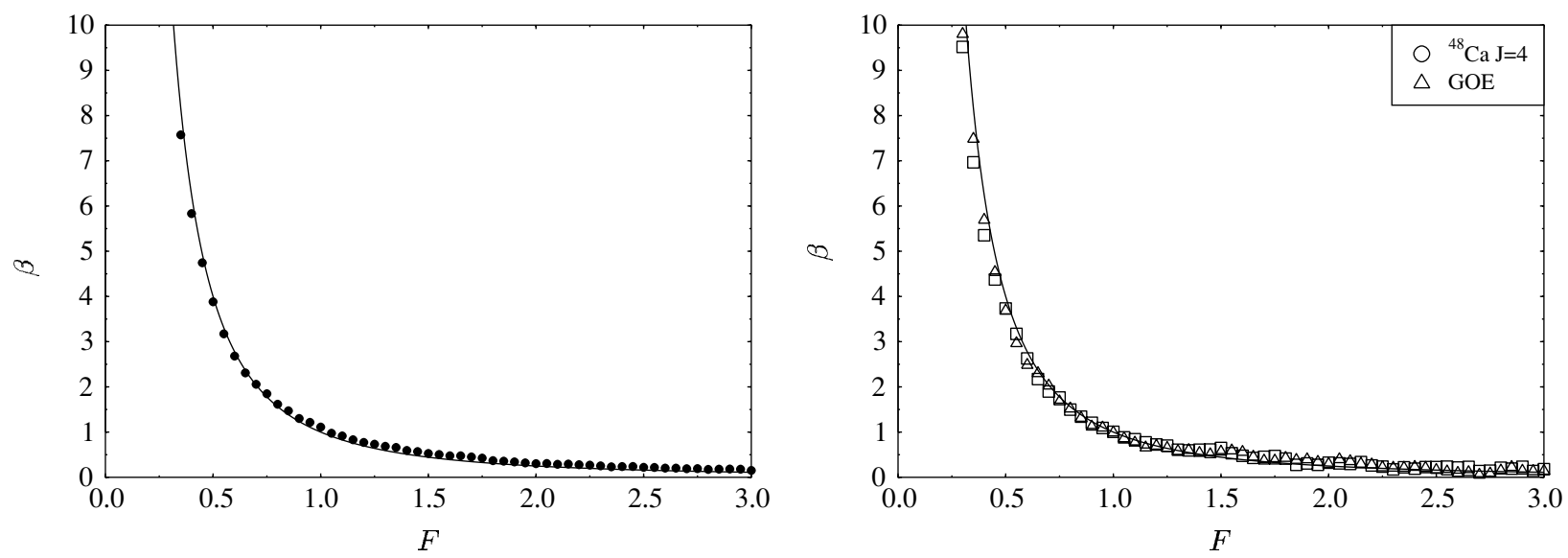

FIG. 4. The repulsion parameter $\beta$ in Eq. (4) as a function of $F$. Left-hand panel: results for an ensemble average of 100 matrices of the spin model explained in the text. Right-hand panel: results for a shell-model matrix for the ${ }^{48} \mathrm{Ca}, J=4^{+}$states in the $p f$ shell [18] and for a single GOE matrix of dimension $d=1000$. The errors in the fitted value of $\beta$ are given approximately by the size of the symbols. They are smaller in the left-hand panel because of the ensemble average.

where the first equality is $s_{i}$ by definition, and it takes a little geometry to check the second equality, i.e., the standard prescription that we have followed relying on Eq. (12). Let us compare now the Brody and Izrailev parametrizations of $P(s, \beta)$ for ensemble averages of the spin system at $F=0.25$ and $F=2$ in Fig. 3. For $F=2$, and in general for $F>1$, the form of both distributions and the value of $\beta$ are almost the same and the fit is very good. For $F<1$ the values of $\beta$ become different, and Izrailev's prescription is much closer to the Wigner surmises [Eq. (1)] and accounts better for the behavior at large and small spacings. Therefore we adopt it from now on.



FIG. 5. The $\bar{\Delta}_{3}(L)$ statistic for different values of the amplitude of matrix fluctuations $F$ for the system of interacting spins. Diamonds: $F=3, \beta=0.17$; the result is close to Poisson. Filled circles: $F=1, \beta=1.0$, and the result coincides with GOE. Squares: $F=0.75, \beta=1.9$, and results are near the GUE curve. Triangles: $F=0.5, \beta=3.87$ are very near the GSE result (dashed-dotted line). For $F=0$, the picket-fence spectrum $\Delta_{3}(L)$ (open circles) is constant and equal to $1 / 12$.

\section{UNIVERSALITY OF FLUCTUATION PROPERTIES}

To establish the general validity of Eq. (7), we show results of three different systems, a GOE matrix, the Hamiltonian of $N$ spins of Eq. (8), and a shell-model matrix for the states $J=4^{+}$ of ${ }^{48} \mathrm{Ca}$ [18]. This specific case was chosen for presentation because its dimensionality, $d=1755$, makes it convenient for statistical studies, but we have checked many other examples, always with the same results.

In Fig. 4 the behavior of $\beta$ as a function of $F$ in Eq. (6) is shown for ensemble averages of the spin model (parameters are as in Fig. 1) and for single GOE and ${ }^{48} \mathrm{Ca}$ matrices. It is clear that the results are perfectly reproduced in the three cases by expression (7). It appears that fluctuation properties in any particular case are dictated by the amplitude of the fluctuations in a single tridiagonal matrix. The results for $\bar{\Delta}_{3}(L)$, shown in Fig. 5 for a few selected cases of a typical realization, corroborate this conjecture. It is remarkable that we can explore all possible values of the repulsion parameter $\beta$, from 0 to $\infty$, as a function of the parameter $F$. Both the short-range correlations in the spectra, as shown by the results for the $P(s)$, and the long-range ones, as shown by the calculation of the $\Delta_{3}$, coincide with the prediction of the RMT when $\beta$ takes the values corresponding to the classical ensembles.

The form of Eq. (7) is somewhat artificial and open to three objections: (a) its simplicity is due to an accident because for the chosen examples $F=1$ corresponds to $\beta=1$; (b) it is not strictly exact; and (c) it does not relate $\beta$ to quantities directly associated with a given Hamilonian. Therefore, because $F$ is obviously proportional to the width of the fluctuations $\sigma_{f}$, we redefine $F=\varkappa \sigma_{f} / \sigma$, where $\sigma$ is the width of the Hamiltonian. Ensemble averages for matrices of dimensionality $d=50$ to 800 yield the following fit:

$$
\beta^{\alpha} \approx \frac{1}{2 d} \frac{\sigma^{2}}{\sigma_{f}^{2}}=\frac{1}{F^{2}}, \quad \alpha=1.08 \pm 0.04
$$




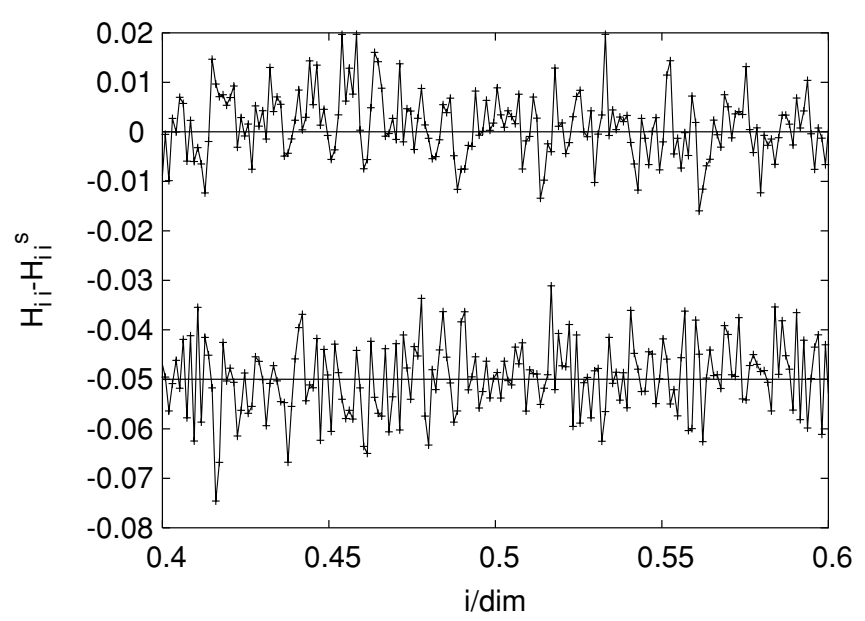

FIG. 6. The fluctuating diagonal contribution for a spin and a GOE matrix (below, displaced by -0.05 units). There are 100 changes of sign for GOE, against 76 in the spin case, for a total of 184 levels around the centroid. $\operatorname{dim}=924$.

a useful relation that defines $\varkappa$ and suggests $\beta \approx F^{-1.9}$, which fits Fig. 4 slightly better than $\beta=F^{-2}$.

Nothing general can be said about the correlations of the tridiagonal fluctuations, which depend on the Hamiltonian. For GOE matrices they can be assimilated to white noise: The distribution is normal, and no correlations are detected. For the spin matrices we can say that their fluctuations are definitely not white noise and that the subject deserves further study. Analyses in terms of $1 / f$ noise [19] may be of interest in this respect. Figure 6 gives some idea of the different behavior in both cases: There is more correlation between neighboring spin matrix elements, reflected in less-frequent changes in sign. It follows that the universal behavior detected in Eq. (7) is not related to the type of correlations in the tridiagonal fluctuations. Therefore it must be viewed as a general property emerging from the diagonalization process itself. This result is crucial as it explains why $P(s, \beta)$ is such a robust feature of numerical simulations and why it contains little information about specific systems. Such information is to be found in the tridiagonal matrices, not in the spectrum.

\section{CONCLUDING REMARKS}

The simplicty of the form of Eq. (7) is due to an accident because, for the chosen examples, $F=1$ corresponds to $\beta=1$. In the case of GOE the correspondence is natural. For the spin and shell-model matrices it is related to the special status of the GOE Wigner surmise: Physical systems do not seem prone to deviate from it. In other words, in $H=H_{d}+\lambda V$ studies it usually takes a small $\lambda$ to move to the Wigner limit. This is a fascinating subject outside the scope of this study. A challenging question remains: As the extraction of $\beta$ is a tedious operation, why not use $F$ directly as a parameter? We hope soon to provide an answer.

\section{ACKNOWLEDGMENTS}

We thank Oriol Bohigas for enlightening discussions. This work is supported in part by Spanish government grants BFM2000-0600 and FTN2000-0963-C02. R. A. Molina acknowledges financial support from the European Uniońs Human Potential Program (contract no. HPRN-CT-200000144).
[1] C. E. Porter, ed., Statistical Theories of Spectra: Fluctuations (Academic Press, New York, 1965).

[2] T. A. Brody, J. Flores, J. B. French, P. A. Mello, A. Pandey, and S. S. M. Wong, Rev. Mod. Phys. 53, 385 (1981).

[3] K. K. Mon and J. B. French, Ann. Phys. (NY) 95, 90 (1975).

[4] F. M. Izrailev, Phys. Rep. 196, 299 (1990).

[5] V. Zelevinsky, B. A. Brown, N. Frazier, and M. Horoi, Phys. Rep. 276, 85 (1996).

[6] G. P. Berman, F. Borgonovi, F. M. Izrailev, and V. I. Tsifrinovich, Phys. Rev. E 65, 015204(R) (2001).

[7] M. V. Berry and M. Tabor, Proc. R. Ser. London Sec. A 356, 375 (1977).

[8] T. A. Brody, Lett. Nuovo Cimento 7, 482 (1973).

[9] F. M. Izrailev, Phys. Lett. A134, 13 (1988).

[10] F. J. Dyson, J. Math. Phys. 3, 140 (1962).
[11] R. U. Haq, A. Pandey, and O. Bohigas, Phys. Rev. Lett. 48, 1086 (1982).

[12] M. S. Bae, T. Otsuka, T. Mizusaki, and N. Fukunishi, Phys. Rev. Lett. 69, 2349 (1992).

[13] R. A. Molina, J. M. G. Gómez, and J. Retamosa, Phys. Rev. C 63, 014311 (2001).

[14] A. P. Zuker, L. Waha-Ndeuna, F. Nowacki, and E. Caurier, Phys. Rev. C 64, 021304(R) (2001).

[15] A. P. Zuker, Phys. Rev. C 64, 021303(R) (2001).

[16] A. D. Jackson, C. Mejia-Monasterio, T. Rupp, M. Saltzer, and T. Wilke, Nucl. Phys. A687, 405 (2001).

[17] J. M. G. Gómez, R. A. Molina, A. Relãno, and J. Retamosa, Phys. Rev. E 66, 036209 (2002).

[18] E. Caurier, A. P. Zuker, A. Poves, and G. Martínez-Pinedo, Phys. Rev. C 50, 225 (1994).

[19] A. Relãno, J. M. G. Gómez, R. A. Molina, J. Retamosa, and E. Faleiro, Phys. Rev. Lett. 89, 244102 (2002). 